\title{
Acquisizione linguistica precoce e ruolo dell'adulto: la formazione in servizio come occasione di crescita professionale
}

\author{
Sergio Crasnich \\ Istituto dell'Educazione della Repubblica di Slovenia \\ sergio.crasnich@zrss.si
}

\begin{abstract}
L'introduzione precoce all'acquisizione di una seconda lingua rappresenta una straordinaria opportunità in contesti etnicamente e linguisticamente misti. Una strategia per superare i vincoli posti dalla condizione di mancata padronanza, da parte del bambino, della lingua utilizzata nella scuola dell'infanzia è offerta dal ricorso a metodi e tecniche riconducibili all'approccio umanistico alla didattica della lingua. Il contributo presenta un percorso di formazione in servizio, che ha coinvolto educatori ed educatrici delle scuole dell'infanzia con lingua d'insegnamento italiana sul territorio nazionalmente misto dell'Istria slovena. Le attività dei partecipanti, realizzate in un contesto ispirato ai principi della ricerca-azione e dell'apprendimento cooperativo, si sono proposte di individuare elementi di connessione tra le indicazioni del Programma curricolare sloveno per le scuole dell'infanzia, alcune proposte glottodidattiche legate alla pedagogia della comunicazione verbale, e talune modalità di organizzazione delle attività formative, già poste in essere dal personale educativo.
\end{abstract}

Keywords: approccio naturale, formazione in servizio, scuole dell'infanzia, lingua italiana, Istria slovena

\section{Introduzione}

L'attuale riflessione pedagogica incoraggia l'accostamento, a partire dalle primissime fasi della vita, a un ventaglio di esperienze educative e formative il più ampio e il meglio calibrato possibile. Obiettivo di tale precoce stimolazione è di consentire lo sviluppo epigenetico di connessioni e reti neurali, in un percorso che considera ogni essere umano come un'entità unica e irripetibile, il cui farsi è espressione al tempo stesso del patrimonio genetico e delle esperienze ambientali. L'opportunità di predisporre contesti ottimali di stimolazione delle competenze del bambino assume cruciale importanza nel caso dell'acquisizione della lingua (Daloiso 2009). I più recenti studi sul bilinguismo hanno evidenziato che l'esposizione precoce a una lingua non materna produce effetti di competenza praticamente indistinguibili da quelli osservati su parlanti madrelingua, sia dal punto di vista morfosintattico e 
lessicale sia per quanto riguarda la pronuncia. Anche a livello di fluidità del pensiero, la prestazione del bilingue precoce è diversa da quella del bilingue esposto a una lingua in fase evolutiva successiva, poiché presenta un carattere di maggiore immediatezza e non passa attraverso il filtro della lingua materna (Balboni 2015). L'esposizione precoce a una seconda lingua costituisce una irrinunciabile opportunità in contesti etnicamente e linguisticamente misti, quali quello dell'Istria slovena, nel quale la lingua italiana costituisce per buona parte della popolazione una lingua seconda.

\section{Sviluppo linguistico e bilinguismo precoce: la specificità del contesto italiano dell'Istria slovena}

Il Programma curricolare per le scuole per l'infanzia, pur indicando esplicitamente nel bilinguismo la meta cui deve tendere l'impegno educativo e didattico nei territori etnicamente e linguisticamente misti (Ministero dell'Istruzione e dello Sport 2000, 8) non fornisce indicazioni specifiche in merito alle modalità di azione nei casi in cui la lingua materna del bambino non coincida con quella utilizzata all'interno dell'istituzione educativa. Quest'ultima circostanza emerge con particolare salienza nelle istituzioni educative con lingua d'insegnamento italiana sul territorio nazionalmente misto dell'Istria slovena.

A titolo di esemplificazione, saranno presentati i dati generali riguardanti gli anni 1999, 2010 e 2017, raccolti da parte dell'Istituto dell'educazione della Repubblica di Slovenia. Nel 1999 il numero complessivo di alunni era di 1020: di questi, gli iscritti alla scuola dell'infanzia corrispondevano al $23 \%$, quelli iscritti alla scuola elementare al $49 \%$, quelli iscritti alla scuola media al $28 \%$. $\mathrm{Nel} 2010$ il numero complessivo di alunni era di 983: di questi, gli iscritti alla scuola dell'infanzia corrispondevano al $44 \%$, quelli iscritti alla scuola elementare al 41\%, quelli iscritti alla scuola media al 15\%. Nel 2017 il numero complessivo di alunni era di 1216: di questi, gli iscritti alla scuola dell'infanzia corrispondevano al $42 \%$, quelli iscritti alla scuola elementare al $46 \%$, quelli iscritti alla scuola media al $12 \%$. Come si può osservare, accanto a un rilevante aumento generale del numero di alunni negli anni dal 2010 al 2017, va segnalato il marcato aumento proporzionale degli iscritti alle scuole dell'infanzia, che sono più che raddoppiati passando da circa un quinto (nel 1999) a oltre due quinti della popolazione scolastica complessiva (sia nel 2010, sia nel 2017).

La provenienza linguistica degli alunni frequentanti le istituzioni scolastiche con lingua d'insegnamento italiana sul territorio nazionalmente misto dell'Istria slovena non è rilevata al momento della loro iscrizione, né è finora divenuta oggetto di ricerca. Tuttavia, nell'ambito delle attività di formazione 
in servizio coordinate dall'Istituto dell'educazione della Repubblica di Slovenia sono state condotte alcune indagini esplorative (Crasnich 2014), grazie alle quali è possibile tratteggiare un quadro che comprende almeno le seguenti categorie: (1) monolingui italofoni (a casa parlano solamente l'italiano); (2) bilingui (a casa parlano l'italiano e lo sloveno/il croato); (3) bilingui (a casa parlano l'italiano e un'altra lingua delle repubbliche della ex Jugoslavia/di immigrazione); (4) bilingui (a casa parlano lo sloveno/il croato e un'altra lingua delle repubbliche della ex Jugoslavia/di immigrazione); (5) bilingui (a casa parlano due lingue delle repubbliche della ex Jugoslavia/di immigrazione); (6) monolingui non italofoni (a casa parlano lo sloveno/il croato/una lingua di immigrazione); (7) monolingui non italofoni (a casa parlano una lingua delle repubbliche della ex Jugoslavia/una lingua di immigrazione); (8) altre tipologie, legate ai flussi migratori che hanno recentemente interessato la Repubblica di Slovenia, con particolare riguardo all'area del Litorale.

L'introduzione precoce all'acquisizione della lingua italiana da parte di bambini e bambine non italofoni si impone dunque come un impegno inderogabile, cui il personale educativo operante nelle istituzioni minoritarie di lingua italiana deve essere tecnicamente e professionalmente preparato.

\section{Approccio glottodidattico umanistico e scuola dell'infanzia: le ragioni di una sinergia}

Una possibile occasione di soddisfacimento dei vincoli posti dalla condizione di non garantita padronanza, da parte del bambino, della lingua utilizzata all'interno delle istituzioni scolastiche è offerta dal ricorso a modalità, tecniche e metodi riconducibili all'approccio umanistico alla didattica della lingua. Obiettivo comune alle diverse proposte operative formulate da questo approccio (Pichiassi 1999; Caon 2005; Balboni 2015) è di individuare percorsi e strategie in grado di minimizzare ostacoli e resistenze di natura psicoaffettiva, che esercitano effetti particolarmente evidenti se la lingua utilizzata non è ancora pienamente padroneggiata. Tale opzione trova fondamento e giustificazione in istanze proprie della pedagogia umanistica (Rogers 1951; 1969) di cui si propone il trasferimento in ambito glottodidattico.

I presupposti teorici alla base dell'approccio umanistico muovono da un'intenzionale azione di confronto con le condizioni che promuovono l'acquisizione della lingua in contesti naturali. Nella teoria di Krashen e Terrell (1983), che gli autori stessi definiscono approccio naturale (natural approach), la quantità di input linguistico offerto è più importante della pratica, ed è fondamentale attendere il momento in cui l'apprendente inizierà a usare 
spontaneamente la lingua obiettivo, senza richiedere prestazioni premature e suscettibili di dare luogo a inibizione.

In condizioni spontanee, la lingua viene acquisita seguendo un percorso a spirale, che procede per approssimazioni e fasi di interlingua (Selinker 1992) oggetto di studio della linguistica acquisizionale (Chini 2005): in sostanza, da una iniziale semplificazione si procede alla risistemazione e all'incremento in quantità e qualità delle proprie competenze, in una successione di configurazioni uniche, originali, soggette a costante ristrutturazione. II passaggio da una configurazione all'altra è promosso dal costante arricchimento qualitativo dei messaggi indirizzati alla persona, che costituiscono l'input da comprendere. Un input è comprensibile se si colloca su un gradino immediatamente successivo a quello che caratterizza il livello di competenza già padroneggiato dall'ascoltatore: inoltre, se il parlante fornisce un sistema di supporto all'acquisizione del linguaggio (LASS, Language Acquisition Support System) i processi automatici di comprensione facenti capo al meccanismo di acquisizione della lingua (LAD, Language Acquisition Device) si attivano autonomamente e operano con efficacia. Krashen e Terrell (1983) sottolineano che l'input comprensibile permette il passaggio graduale da un livello di competenza meno articolato a un livello più articolato; i riferimenti psicopedagogici cui questo principio fa appello sono costituiti dall'area di sviluppo potenziale di Vygotskij e dalla zona di sviluppo prossimale di Bruner, nonché dalla metafora bruneriana dello scaffolding, costituito in questo caso dalle enunciazioni del parlante più esperto.

\section{La ricerca: contesto, partecipanti, finalità, obiettivi, metodo e procedura}

Il presente lavoro nasce da una richiesta esplicitamente rivolta all'autore (nel suo ruolo di consulente pedagogico) da parte del personale educativo, mirante a problematizzare due questioni: (1) la qualità dell'offerta formativa delle istituzioni rispetto sia alla normativa vigente, sia alla più recente, aperta e illuminata riflessione pedagogica, didattica ed educativa; (2) la qualità dell'input offerto dal personale educativo, rispetto all'esigenza di garantire le condizioni ottimali per il potenziamento delle competenze linguistiche dei bambini. Tale richiesta ha costituito il riferimento al quale sono state vincolate tutte le scelte di disegno adottate per l'intervento.

Alla ricerca ha partecipato un gruppo di venti educatrici ed educatori. L'intervento si è svolto nell'ambito delle attività di formazione in servizio organizzate dall'Istituto dell'educazione della Repubblica di Slovenia.

La ricerca, avente a oggetto le modalità di organizzazione dell'offerta formativa, si è posta la finalità di favorire l'integrazione tra queste ultime, defi- 
nite dal vigente Programma curricolare, e alcune proposte di buone pratiche legate alla glottodidattica umanistica.

Gli obiettivi della ricerca, sottesi dalla richiesta di intervento formativo, sono stati i seguenti:

1. individuare sinergie tra le modalità di organizzazione dell'offerta formativa delle scuole e le più rilevanti proposte della glottodidattica umanistica;

2. incoraggiare la riflessione dei partecipanti su alcune proposte della glottodidattica umanistica, in modo da promuoverne l'implementazione.

Gli interrogativi di ricerca che ci si è posti sono i seguenti:

1. È possibile individuare sinergie tra le modalità di organizzazione dell'offerta formativa delle scuole e alcune proposte della glottodidattica umanistica?

2. Ė possibile incoraggiare la riflessione dei partecipanti su alcune proposte della glottodidattica umanistica, in modo da promuoverne l'implementazione?

Il metodo di ricerca adottato si è ispirato all'approccio della ricerca-azione (De Landsheere 1982; Elliott, Giordan e Scurati 1993; Barbier 2007), considerato la modalità più idonea a favorire percorsi partecipativi di acquisizione di conoscenza, riflessione e promozione del cambiamento.

Per quanto riguarda la procedura attuata, il formatore ha diviso l'intervento in due parti (una per ciascuna delle questioni problematizzate), con incontri articolati in tre fasi: (a) una fase introduttiva dedicata alla presentazione della tematica da trattare; (b) una fase laboratoriale, in cui i partecipanti erano invitati a svolgere attività di lettura, confronto e riflessione secondo le modalità dell'apprendimento cooperativo (Comoglio e Cardoso 1996); (c) una fase di restituzione delle riflessioni dei gruppi, con l'individuazione di possibili punti di condivisione. Nella prima fase degli incontri, la condotta comunicativa del formatore è stata improntata all'uso di modalità discorsive basate sulla spiegazione, supportata dalla presentazione di diapositive in formato Powerpoint. Nella seconda e terza fase, invece, la condotta comunicativa è stata improntata all'adozione di interventi di risposta a riflesso o riformulazione a specchio (Rogers 1951), allo scopo di incoraggiare la verbalizzazione di idee, proposte e riflessioni da parte dei gruppi. Solo a verbalizzazione pienamente conclusa, il formatore ha proposto delle ricapitolazioni, miranti a sintetizzare i punti condivisi. 


\section{Risultati della ricerca e discussione}

Nei due successivi paragrafi saranno descritti gli esiti più salienti della prima e della seconda parte dell'intervento. Nella discussione si mostrerà che le riflessioni emerse nel corso delle due parti dell'intervento hanno permesso ai partecipanti: (1) di individuare sinergie tra le modalità di organizzazione dell'offerta formativa delle scuole dell'infanzia e le più rilevanti proposte della glottodidattica umanistica; (2) di riconoscere la validità di alcune proposte di intervento della glottodidattica umanistica, come condizione per promuoverne una successiva implementazione. Di conseguenza, si ritiene di poter dare risposta positiva a entrambi gli interrogativi che la ricerca si è proposta.

\section{Prima parte dell'intervento: i requisiti di un educatore umanistico}

Nella prima parte dell'attività formativa sono stati presentati gli elementi salienti dell'approccio naturale e della pedagogia umanistica, e si è proposto di riflettere criticamente sulle indicazioni del Programma curricolare per la scuola dell'infanzia (Ministero dell'Istruzione e dello Sport 2000), allo scopo di individuare il profilo di un educatore orientato in senso umanistico. L'attività del personale educativo coinvolto nell'iniziativa, incoraggiata e facilitata dal formatore, ha portato all'individuazione di quattro requisiti. Questi ultimi saranno ora presentati in riferimento alla letteratura scientifica che li supporta, e accompagnati dalla citazione dei passi del Programma curricolare per le scuole dell'infanzia cui risultano riconducibili.

II primo requisito è stato sintetizzato come la capacità di creare un ambiente caldo, supportivo, incoraggiante. II riferimento curricolare da esso sotteso (Ministero dell'Istruzione e dello Sport 2000, 39) sottolinea l'importanza di creare un'atmosfera tale che spinga il bambino a parlare, ad allargare la sua esperienza del discorso, ad esprimere i suoi sentimenti, desideri e bisogni. Di fondamentale importanza per l'acquisizione di questa capacità è stata giudicata la presenza, all'interno della sezione, del clima di ascolto attivo e incondizionato che la psicologia e la pedagogia umanistiche considerano come condizione di base per la promozione di qualsiasi forma di dialogo educativo (Kanizsa 2007); a questi elementi fa esplicito riferimento anche il documento curricolare, quando rimarca l'impegno dell'insegnante a creare un clima piacevole, di fiducia reciproca, nel quale il bambino venga sollecitato alla comunicazione, all'espressione, alla partecipazione e alla creatività (Ministero dell'Istruzione e dello Sport 2000, 54).

II secondo requisito è stato sintetizzato come la capacità di osservare i bambini per coglierne le diversità e reagire empaticamente, usando la comunicazione in tutte le sue sfaccettature. II riferimento curricolare da esso sotteso (Mini- 
stero dell'Istruzione e dello Sport 2000, 38) vede nell'osservazione da parte dell'adulto una condizione necessaria e preliminare per individuare le capacità comunicative dei bambini (Mantovani 1995; Reffieuna 2002), cercando di comprendere i messaggi espressi non verbalmente, come il linguaggio corporeo. Importante è anche la capacità di rispondere al bambino utilizzando il linguaggio corporeo e della comunicazione non verbale (Bonaiuto e Maricchiolo 2009), che assume particolare importanza quando il bambino, a causa dell'età, non dispone ancora di un grande patrimonio lessicale o quando desidera esprimere contenuti molto intimi, nascosti e difficilmente esprimibili con le parole (Ministero dell'Istruzione e dello Sport 2000, 55).

II terzo requisito è stato sintetizzato come la capacità di rispettare l'individualità del bambino, nei suoi stili ma anche nei suoi silenzi o nelle sue reticenze. Il riferimento curricolare da esso sotteso riguarda la necessità di adeguare le richieste allo sviluppo evolutivo del bambino e alle sue capacità discorsive, rispettando le sue modalità di interazione con l'ambiente e stimolando all'espressione dei sentimenti, prima in modo non verbale, in seguito combinando mezzi verbali e non verbali (Ministero dell'Istruzione e dello Sport 2000, 40). Inoltre, è stata considerata fondamentale l'esigenza di rispettare il principio alla riservatezza e all'intimità, che consente al bambino di non esprimere contenuti che preferisce tenere per sé (Ministero dell'Istruzione e dello Sport $2000,11)$. Congruenti con questo requisito curricolare sono state considerate le indicazioni glottodidattiche umanistiche riguardanti la gradualità nella richiesta di prestazioni linguistiche e l'importanza di rispettare la fase del silenzio: quest'ultima è considerata da alcuni autori (Pichiassi 1999; Balboni 2015) caratteristica del momento ricettivo propedeutico alla manifestazione di competenza linguistica produttiva.

Il quarto requisito è stato sintetizzato come la capacità di accettazione incondizionata e di riformulazione della comunicazione. L'incoraggiamento della comunicazione dell'interlocutore è stato oggetto di attenzione e riflessione da parte di autori quali Lumbelli (Lumbelli 1994; Lumbelli 2009) e Pontecorvo (Pontecorvo, Ajello e Zucchermaglio 1991; Pontecorvo 2005), che hanno individuato nella risposta a riflesso o riformulazione a specchio (Rogers 1951) la sua più proficua modalità di implementazione in ambito pedagogico e didattico. II principale vantaggio di tale atto comunicativo consiste nella sua efficacia nel fornire al parlante un feedback non valutativo, che promuove la prosecuzione della comunicazione e la verbalizzazione dei processi di pensiero. A esso pare riferirsi direttamente anche il Programma curricolare per le scuole dell'infanzia, quando rimarca la necessità di seguire con attenzione le conversazioni tra bambini, cercando di riprendere e approfondire gli argo- 
menti spontaneamente proposti da questi ultimi (Ministero dell'Istruzione e dello Sport 2000, 39), nonché di stimolare il bambino nei primi tentativi di verbalizzazione, ripetendo e facendo eco alle sue espressioni (p. 40).

L'applicazione dei requisiti fin qui individuati e riassunti dovrebbe permeare la globalità dell'azione educativa e didattica dell'adulto, attuandosi per mezzo di specifiche modalità di interazione verbale. In questo senso, assume decisiva importanza la capacità dell'adulto di fornire al bambino un sistema di supporto all'acquisizione linguistica sotto forma di input ampio, differenziato, comprensibile perché contestualizzato, in grado di attivare $\mathrm{i}$ meccanismi spontanei di acquisizione.

\section{Seconda parte dell'intervento: l'interazione verbale di un educatore umanistico}

Nella seconda parte dell'attività formativa, ai partecipanti è stato proposto di riflettere criticamente sulle proprie modalità comunicative allo scopo di individuare congruenze o incongruenze con il profilo dell'educatore umanistico precedentemente tratteggiato. L'attività del personale educativo coinvolto nell'iniziativa, anche in questo caso incoraggiata e facilitata dal formatore nei momenti di lavoro dei gruppi, ha portato all'individuazione di condotte verbali, suscettibili di arricchimento e potenziale influsso positivo sulle competenze linguistiche dei bambini.

Al personale educativo sono state proposte le consegne sottostanti:

1. Dividetevi in gruppi omogenei, all'interno dei quali siano presenti educatori, educatrici, aiuto-educatori e aiuto-educatrici.

2. Ogni gruppo scelga una fascia di età, corrispondente a quelle presenti all'interno della scuola dell'infanzia.

3. Discutendo e riflettendo su quanto previsto dal Programma curricolare per le scuole dell'infanzia, ogni gruppo individui i nuclei di attività implementati nella programmazione didattica.

4. Per ciascun nucleo di attività, ogni gruppo individui e trascriva le parole, espressioni o frasi solitamente utilizzate per comunicare con i bambini.

5. Discutendo e riflettendo su quanto emerso dal lavoro dei gruppi, il collettivo confronti le parole, espressioni o frasi trascritte e ne analizzi la struttura, proponendo eventuali integrazioni o modifiche.

6. Al termine, il collettivo dovrebbe disporre di un protocollo minimo, composto da parole, espressioni o frasi condivise da tutti gli operatori, e la cui struttura è stata analizzata e ottimizzata. 
Di seguito si propone la trascrizione degli enunciati proposti dagli educatori operanti con la prima fascia di età (da zero a tre anni).

Accoglienza

Buongiorno (nome del bambino), ben arrivato

Come stai?

Giochi liberi/attività

Vieni al tavolo

Guarda che bel libro

Mostrami cosa hai portato

Merenda

Riordiniamo i giochi e le sedie

Andiamo a lavarci le mani

Rimboccatevi le maniche

Apri il rubinetto

Poca acqua

Poco sapone

Sciacqua bene le mani

Chiudi il rubinetto

Prendi una carta (asciugamani) e asciugati le mani

Il pasto

Vai a sedere al tuo posto

Buon appetito

Grazie

Prego

Attività

Andate a sedere nell'angolino morbido/Sedetevi nell'angolino morbido

Ripetiamo/Ripeteremo le canzoncine imparate

E adesso il girotondo ... diamoci la mano, prendetevi per la mano

Riposo

Vieni andiamo a cambiare il pannolino

Mettiamo la crema

Dove il tuo pannolino

E adesso sul proprio lettino/sulla brandina e buon riposo

Risveglio

Ben svegliati 
Prendi le pantofole/le ciabatte

Dammi il ciuccio

\section{Congedo}

Arrivederci a domani

Ci vediamo domani

L'intervento di riflessione e confronto, sviluppato dal personale educativo con la mediazione del formatore, ha permesso di individuare una serie di aspetti critici.

Le attività si organizzano intorno ai momenti previsti dal Programma curricolare per le scuole dell'infanzia (Ministero dell'Istruzione e dello Sport 2000); tuttavia, non sembrano essere sufficientemente valorizzati i momenti dedicati al dialogo, allo scambio comunicativo, alla condivisione di esperienze. L'interazione educativa che emerge dalle enunciazioni rievocate dal gruppo evidenza vicinanza ed empatia, ma non assume come momento centrale la stimolazione, l'incoraggiamento, il potenziamento della comunicazione e del dialogo. Vi è indubbiamente cordialità (si vedano per esempio le formule relative ai momenti dell'accoglienza e del congedo) ma le formule utilizzate sono semplici e stereotipate: non presentano, quindi, quelle caratteristiche di ridondanza, ricchezza e naturalità attribuite a un input che, in quanto accompagnato da elementi non verbali, sarebbe comunque comprensibile ai bambini e si situerebbe all'interno della loro area di sviluppo potenziale.

Un altro commento riguarda la sottolineatura linguistica che accompagna i momenti di attività situati tra l'accoglienza e il congedo. Prevalgono brevi enunciati di tipo regolativo, il più delle volte proposti con l'uso della seconda persona singolare o plurale dell'imperativo (vieni ..., rimboccatevi ..., apri ..., sciacqua ..., chiudi ..., prendi ..., vai a sedere ..., andate a sedere/sedetevi ..., prendetevi ..., prendi ..., dammi ...). Meno marcata, invece, risulta la presenza della prima persona plurale del congiuntivo che, oltre ad assumere funzione esortativa, comunica al destinatario del messaggio la partecipazione e la vicinanza dell'emittente (riordiniamo ..., andiamo a lavarci ..., ripetiamo ..., diamoci la mano ..., andiamo a cambiare ..., mettiamo...).

Negli enunciati sono poi documentati esempi di frasi ellittiche (poca acqua ..., poco sapone ..., Dove il tuo pannolino ..., E adesso sul proprio lettino/sulla brandina), la cui comprensione è probabilmente garantita dal contesto extralinguistico all'interno delle quali sono collocate. Tuttavia, nell'ottica di favorire e accompagnare l'acquisizione naturale della lingua, esse potrebbero essere seguite da ulteriori riformulazioni complete, che sarebbero age- 
volmente processabili dai bambini (Pienemann 1998) dato il contesto, noto e quotidianamente condiviso, in cui ha luogo l'interazione con l'adulto.

A seguito della riflessione di cui si sono ora sintetizzati i punti salienti, è stato chiesto ai partecipanti di immaginare delle modifiche della propria condotta linguistica, miranti ad associare a tutte le attività delle enunciazioni e spiegazioni di carattere integrativo (Lumbelli 1989). Esse, accompagnando la scansione delle azioni e dei gesti compiuti dall'adulto o dai bambini, potrebbero creare le precondizioni per favorire il passaggio dall'input all'intake. $\mathrm{Di}$ seguito si propone l'esempio di una delle riformulazioni operate dal gruppo e rivista dal formatore, riguardante il momento dell'accoglienza mattutina: in essa, la condotta linguistica dell'adulto punteggia e sottolinea le azioni che il bambino compie, da solo o aiutato.

Buongiorno, ben arrivato. Come stai? Hai dormito bene? Vieni, andiamo a togliere il cappotto. Ecco, mettiamolo sull'appendiabiti ... così. Ora togliamo le scarpe e mettiamo le pantofole ... togliamo la scarpa destra ... ecco ... adesso invece togliamo la scarpa sinistra ... bene ... mettiamo le scarpe nella mensola che sta sotto il cappotto ... Benissimo, adesso siamo pronti per andare nella nostra aula ... Salutiamo la mamma ... Se vuoi, puoi darmi la mano... Andiamo ... adesso andremo a giocare per un po' con il gioco che preferisci ...

La riscrittura riprende gli aspetti topici del momento dell'accoglienza, e l'adulto accompagna l'esecuzione di azioni ben note al bambino poiché legate alle routine quotidiane. Le parole e le espressioni introdotte negli enunciati (cappotto, appendiabiti, scarpe, pantofole, mensola, aula, mano, gioco) arricchiscono l'input lessicale offerto, e la loro processabilità è assicurata dalla presenza dei referenti extralinguistici nell'esperienza condivisa. Alcune locuzioni accompagnano l'acquisizione di modalità descrittive di relazioni reciproche (destra, sinistra, sotto il) importanti per il successivo sviluppo di competenze spaziali e matematiche.

La verbalizzazione sottolinea le dimensioni di autonomia e scelta, che il Programma curricolare per le scuole dell'infanzia (Ministero dell'Istruzione e dello Sport 2000) pone quali linee guida dell'azione educativa (Se vuoi, puoi darmi la mano... con il gioco che preferisci).

L'esempio illustra altresì come, in un contesto autentico, una medesima espressione possa essere ripetuta più volte, e quindi consolidata, senza rischiare di indurre noia (ora togliamo ..., togliamo..., adesso invece togliamo). 
Al tempo stesso, i due momenti dell'azione (ingresso) e della rappresentazione (successiva attività di gioco) sono collocati nelle dimensioni temporali del presente e del futuro attraverso l'uso opportuno dei rispettivi tempi del verbo all'indicativo (andiamo a togliere il cappotto; andremo a giocare).

Infine, la riformulazione propone al bambino parole appartenenti alle principali categorie morfologiche della lingua italiana (articoli, sostantivi, aggettivi, verbi, preposizioni, pronomi, avverbi, congiunzioni) comprese quelle (come gli articoli determinativi e le preposizioni articolate) non presenti nella lingua slovena (il; sull'; le; la; nella).

\section{Conclusioni}

La valutazione del percorso descritto nel presente contributo richiede particolare cautela, date le caratteristiche partecipative dell'approccio in esso adottato (De Landsheere 1982). II più valido indicatore di bontà dell'intervento, infatti, consisterebbe in una modifica, rilevabile tramite osservazione, della condotta verbale del personale educativo, suscettibile di influire sull'acquisizione linguistica dei bambini (Mantovani 1995). Le condizioni in cui l'esperienza è stata realizzata non hanno reso possibile questo tipo di controllo. Tuttavia, essa ha stimolato l'attivazione di un atteggiamento critico e riflessivo, irrinunciabile per la crescita professionale del personale educativo e docente (Cencič 2015).

Un'occasione di controllo empirico delle istanze emerse dalla riflessione degli educatori potrebbe essere costituita dall'approfondimento di alcune problematiche riconducibili alla didattica acquisizionale (Rastelli 2009). In tale prospettiva, un input calibrato in modo da proporre elementi non presenti nella lingua materna del bambino potrebbe favorire la comparsa di enunciati attestanti il raggiungimento di livelli crescenti di competenza. Per esempio, nel caso di un bambino di madrelingua slovena, a una prima fase interlinguistica caratterizzata dall'uso di sostantivi privi di articolo, potrebbe fare seguito una fase in cui una delle forme dell'articolo determinativo viene introdotta senza riguardo alla concordanza di genere e/o numero con il sostantivo che la accompagna (p.e. il piatto, il bicchiere, il tovagliolo, il cucchiaio, il coltello, il forchetta, il bottiglia; il piede, il ginocchio, il gomito, il mano) prima di giungere all'uso corretto dell'articolo determinativo.

Un'ipotesi di lavoro quale quella ora abbozzata assegna all'educatore un ruolo di attiva riflessione sulla propria azione, alle istituzioni universitarie e ai centri di ricerca un ruolo di elaborazione e sperimentazione di metodologie e approcci innovativi, da introdurre nei percorsi di formazione inizia- 
le, e agli istituti di consulenza un ruolo di disseminazione di buone pratiche nell'ambito della formazione in servizio.

\section{Riferimenti bibliografici}

Balboni, P. E. 2015. Le sfide di Babele: insegnare le lingue nelle società complesse. Torino: Utet.

Barbier, R. 2007. La ricerca - azione. Roma: Armando.

Bonaiuto, M., e F. Maricchiolo. 2009. La comunicazione non verbale. Roma: Carocci.

Caon, F. 2005. Un approccio umanistico-affettivo all'insegnamento dell'italiano a non nativi. Venezia: Cafoscarina.

Cencič, M. 2015. Izbrani pristopi k spodbujanju refleksije učiteljev. Koper: Annales.

Chini, M. 2005. Che cos'è la linguistica acquisizionale. Roma: Carocci.

Comoglio, M., e M. A. Cardoso. 1996. Insegnare e apprendere in gruppo. Roma: LAS.

Crasnich, S. 2014. «Težave in izzivi poučevanja manjšinske materinščine (italijanščina).» Predstavljeno na konferenci Jeziki v izobraževanju, Ljubljana, 11. april.

Daloiso, M. 2009. Le lingue straniere nella scuola dell'infanzia: fondamenti di glottodidattica. Torino: Utet.

De Landsheere, G. 1982. La recherche expérimentale en education. Lausanne: UNESCO.

Elliott, J., A. Giordan e C. Scurati. 1993. La ricerca-azione: metodiche, strumenti, casi. Torino: Bollati Boringhieri.

Kanizsa, S. 2007. Il lavoro educativo. Milano: Mondadori.

Krashen, S. D., e T. Terrell. 1983. The Natural Approach: Language Acquisition in the Classroom. Hayward: Alemany.

Lumbelli, L. 1989. Fenomenologia dello scrivere chiaro. Roma: Editori Riuniti.

-1994. Pedagogia della comunicazione verbale. Milano: Franco Angeli.

-2009. La comprensione come problema. Roma e Bari: Laterza.

Mantovani, S., a cura di. 1995. La ricerca sul campo in educazione: i metodi qualitativi. Milano: Mondadori.

Ministero dell'Istruzione e dello Sport. 2000. Programma curricolare perle scuole materne. Ljubljana: Ministero dell'Istruzione e dello Sport.

Pichiassi, M. 1999. Fondamenti di glottodidattica. Perugia: Guerra.

Pienemann, M. 1998. Language Processing and Second Language Development: Processability Theory. Amsterdam: Benjamins.

Pontecorvo, C., a cura di. 2005. Discorso e apprendimento: una proposta per l'autoformazione degli insegnanti. Roma: Carocci.

Pontecorvo C., A. M. Ajello e C. Zucchermaglio. 1991. Discutendo si impara: interazione sociale e conoscenza a scuola. Roma: Carocci.

Rastelli, S. 2009. Che cos'è la didattica acquisizionale. Roma: Carocci. 
Reffieuna, A. 2002. Il bambino a scuola: cosa e come osservare. Roma: Carocci. Rogers, C. R. 1951. Client-Centered Therapy. Chicago: Houghton Mifflin. . 1969. Freedom to Learn. Columbus, OH: Charles E. Merrill. Selinker, L. 1992. Rediscovering Interlanguage. London: Longman.

\section{Zgodnje učenje jezika in vloga odraslega: strokovno izpopolnjevanje kot priložnost profesionalne rasti}

Zgodnje učenje drugega jezika predstavlja izredno priložnost za narodno in jezikovno mešana območja. Uporaba metod in tehnik, ki se navezujejo na humanistični pristop poučevanja jezika, je ena od možnih strategij za premagovanje ovir, na katere otrok naleti zaradi pomanjkljivega znanja jezika, ki se uporablja v vrtcu. Prispevek predstavlja primer strokovnega usposabljanja, v katerem je sodelovala skupina vzgojiteljev in vzgojiteljic vrtcev z italijanskim učnim jezikom na narodnostno mešanem območju slovenske Istre. Pri dejavnostih so bili uporabljeni elementi akcijskega raziskovanja in sodelovalnega učenja. Same dejavnosti udeležencev so se osredotočale in navezovale na smernice Kurikuluma za vrtce, didaktične predloge humanističnega pristopa v povezavi z govornim sporazumevanjem in sporočanjem ter načine organiziranja aktivnosti, ki jih vzgojitelji že uporabljajo.

Ključne besede: naravni pristop, strokovno izpopolnjevanje, predšolska vzgoja, italijanščina, slovenska Istra 